\title{
Computers and Internet for Postgraduate Residents and Medical Students
}

\author{
Vidyashankar $\mathbf{C}^{1}$ \\ ${ }^{1}$ Dr. C. Vidyashankar, Oasis Hospital, PO BOX 1016, Al Ain, Abu Dhabi,UAE.
}

Address for Correspondence: E-mail: vidyashankarc@hotmail.com

\section{Introduction}

Computers have become an essential part of our life, with applications in touching all walks of life. The emergence of Internet has expanded its reach even further, with health care being one of the most important beneficiaries. Having worked in the field of medicine both before and after the use of computers and Internet, the changes I have seen have been dramatic.

\section{The Utility of Computers}

Now, most hospitals and chambers of doctors have computers. The main uses of computers are:

1. Data storage and retrieval - Patient data can be stored safely, in a small space and retrieved rapidly.

2. Practice Management - Doctors in private practice can manage their complete practice transactions using computers; including patient reminders, prescriptions generated through computers that can significantly reduce the chances of errors; billing and report generation.

3. Radiology - Ultrasounds, CT and MRI scans all use computers. Now computers have resulted in 'paperless' films for X-Ray's where the films are in digital formats and can be accessed by the doctors using the computers. Similarly lab reports can be easily accesses using computers.

4. Computer Networks and Hospitals - Interlink of computers is called a computer network. Computer networks are established in hospital for easy transfer and access of patient data, integration of lab and radiology services and for administrative purposes like registration, billing etc.

5. Education - Continuing medical education has been made easy with the advent of Internet with easy access to medical journals, CME etc, making presentations. The complete database of journals is available in the Internet through PUBMED. Medical students have access to large numbers of virtual libraries making learning very easy. Many virtual anatomy, surgery tutorials and electronic books are available. Many medical journals have now published medical student editions.

6. Research - Storage and analysis of patient data, statistical analysis and preparing reports and Presentations has been made easier with the use of Internet with customized software.

7. Internet access.

\section{Buying a Computer/Notebook PC}

Technology is advancing at a rapid pace and what you buy today may get outdated within two years time. Maximum life of a computer will be around five years considering the life of the components and their availability. So, while buying a computer try to get one close to the latest configuration.

1. Keep in mind the processor: RAM (random Access memory) that affects the speed of the computer. A certain minimum RAM, currently 2 GB is necessary to run most software and operating systems.

2. Network card for broadband (high speed) Internet access.

3. Wireless card for wireless Internet access.

4. Adequate hard disk space - currently around 120 GB-200GB.

5. DVD/CD ROM drive for backups.

6. Pens drives for backup of your data.

7. Essential Software -
a. Operating System - Windows or Linux.
b. Software for documents and presentations - like MS Word or Open Office.
c. Antivirus.
d. Soft ware for reading PDF documents - Portable document format is the format in which full text of journals are published. It looks similar to the printed page.
e. Clinic management software for those
f. Camera, speakers and microphone for videoconferencing etc. 


\section{Low Cost Notebooks}

Notebook computers small enough to be carried in your apron pocket have recently been launched (2007). These contain the bare minimum configuration - It is possible to access the Internet, edit documents, and prepare presentations email, games and music. The memory is small 2-8 GB, much below the $120 \mathrm{~GB}-200$ GB available in Desktop PC's and notebooks. If you need a computer for these limited purposes and want to carry it with you, these could be the answer - The cost is around \$400-500. An illustration of the (one of the popular low-cost notebooks) is given in the Figure.

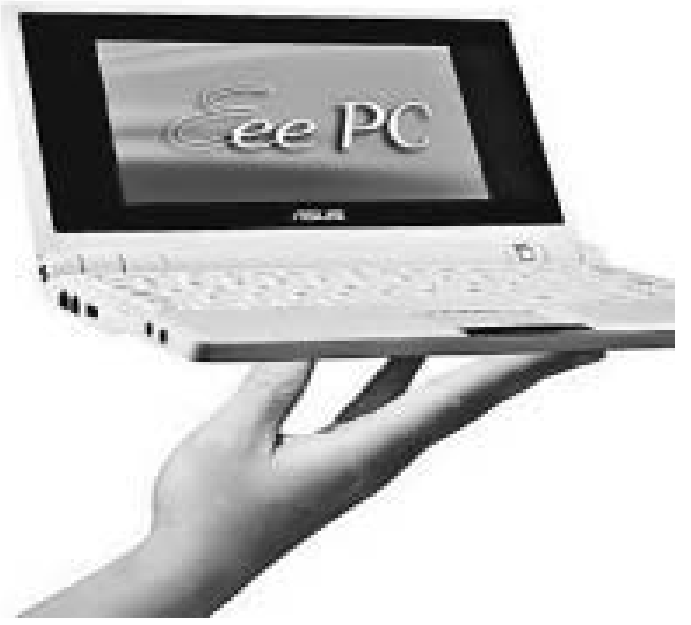

\section{Handheld Computers}

Recent advances in mobile phone technology have resulted in integration of mobile phones with computing powers. These are now freely available with costs reducing. These handhelds can be used for:

1. Email and Internet access (through wireless).

2. Taking notes using the text editor.

3. Electronic textbooks - Most medical textbooks are available in an abbreviated format for handheld computers.

4. Drug doses, formulas for calculating doses of drugs in the PICU and NICU - these are available and can be downloaded.

5. Appointment scheduling.

6. Taking clinical photographs.

7. Take audio/video recordings of lectures, conferences etc.

\section{What you need to know about Internet}

Prior to Internet access to medical journals was possible only in major cities. The most updated information was from recent edition of text books, information which was 1-2 years old. Now recent advances are accessible as soon as the press release is published. From the point of view of students some of the main uses of Internet are:
1. Access medical databases - like Medline/Pubmed which contains abstracts of most of medical journals and HINARI which is a facility of access to full-text journals to developing countries.

2. Access latest editions of textbooks online Updates are available in the Internet much before the print edition.

3. Use for writing dissertation for MD, analysis of data using statistical software.

4. Get latest information through email or RSS (Really Simple Syndication). News feeds or abstracts of news items, including latest issues of medical journals are now delivered to your computer using RSS feeds. This need a RSS reader, which is available free, once installed, subscribe to RSS feeds for the various journals, news websites etc, which are again free. Look for the RSS logo in any website and click for the subscription.

5. Join Bulletin Boards or 'groups' of your interest where you can discuss with other colleagues all over the world.

6. Start a Blog (or a web log) of interesting cases (keep patient identity confidential) so that you can share your experiences with others. Most search engines like Google, MSN offer free Blogs.

7. Telemedicine for consulted with centers in other places - this can help doctors in remote areas transmit their patient data including $X$ ray and images and get a consultation from referral centers.

8. Conference summaries: Most conferences are now webcast that is they can be seen and heard online on our computers through the Internet, which can be accessed from anywhere. You do not have to attend the conferences; the content will be delivered to your PC.

9. Podcasts and Vodcasts: Podcasting is delivering audio content to portable media players (iPods, etc) on demand, so that it can be listened to at the user's convenience. The main benefit of podcasting is that listeners can synchronize content to their media player and take it with them to listen whenever they want to. Because podcasts are typically in popular MP3 audio format, they can also be listened to on nearly any computer. Most medical journals have Podcasts of their major articles. Vodcast are similar, only that the content is video and audio.

10. Online preparation for various entrance exams and career guidance is possible through the Internet. A few of the sites are listed in the references.

11. Prepare patient information leaflets - these can be downloaded from various websites and translated to the local language for distribution to patients. 


\section{Conclusions}

A concise account of the use of computers and Internet for students has been described above. A lot new terminology has been used here. For detailed information on these terms, typing the search term in a search engine like www.google.com will give detailed answers. Technology is changing, so what has been described here may have changed in a short time. What is needed is a regular use of computers and Internet to keep abreast of the recent advances. Computers and Internet have a very important role in improving patient care.

\section{Suggested Reading/Browsing}

Search Engines - www.google.com, www.msn.com, Access Medline - www.pubmed.gov
Free Medical Journals - HINARI - www.who.int/hinari (ask your institutional library for the password!)

Medical News daily - www.medscape.com Medical Student website - www.studentconsult.com Online Textbook - www.emedicine.com Medical Exam preparation - www.rxpg.com, www. aippg.com, www.onexamination.com, Free Medical Statistical Software - EPI Info (www. cdc.gov/epiinfo)

Online Textbooks -MDConsult- www.mdconsult.com 